Scientific Journal of October 6 University

ISSN (Print): 2314-8640

ISSN (Electronic): 2356-8119

Published by October 6 University @ All Rights Reserved

Available online at: http:// sjou.journals.ekb.eg

Original Article
Citation: Abou Zeid et al., (2014). Volatiles Constituents and Anticonvulsant Activity of The Aerial Parts of Dichrostachys Cinerea L. Sci.J. of Oct. 6 Univ. 2(2), 196-200.

Copyright: () 2014 Abou Zeid et al.. This is an open-access article distributed under the terms of the Creative Commons Attribution License, which permits unrestricted use, distribution, and reproduction in any medium, provided the original author and source are credited.

\title{
Volatiles Constituents and Anticonvulsant Activity of the Aerial Parts of Dichrostachys
} Cinerea $\mathbf{L}$

\author{
*Abou Zeid AH ${ }^{1}$, Hifnawy MS ${ }^{2}$, Mohammed RS $^{1}$ and Amany A.Sleem ${ }^{3}$ \\ ${ }^{1}$ Pharmacognosy and ${ }^{3}$ Pharmacology Departments, National Research Centre, El-Tahrir St., Dokki, \\ 12622 , Cairo, Egypt \\ ${ }_{2}^{2}$ Pharmacognosy Department, Faculty of Pharmacy, Cairo University, Kasr Al-Aini St, 11562, Cairo, \\ Egypt
}

Received: 07-03-2014/ Revised: 09-04-2014 / Accepted: 14-04-2014/ published: 01-06-2014

\begin{abstract}
GC/MS analysis of the volatile constituents of aerial parts of $D$. cinerea revealed the identification of 70 compounds representing $(86.13 \%)$ of the total volatiles of the plant. $\alpha$-Pinene $(26.47 \%)$, nonanal $(5.11 \%)$ and 8 , 11,14 eicosatrienoic acid $(4.90 \%)$ were found to be the major compounds. Oxygenated compounds constituted $(32.81 \%)$ mainly attributed to nonanal $(5.11 \%), 8,11,14$ eicosatrienoic acid $(4.90 \%)$, hexadecanoic acid (2.95\%), phytol (1.17\%), $\beta$-ionone $(1.45 \%)$, and methylisoeugenol $(1.08 \%)$. Anticonvulsant activity of the total ethanol extract and successive extracts were investigated which revealed that highest activities were exhibited after 2 hours by $100 \mathrm{mg}$ of total ethanol extract ( $80.87 \%$ potency), methanol extract ( $121.76 \%$ potency) and $100 \mathrm{mg}$ of ethyl acetate extract $(74.15 \%$ potency) respectively.
\end{abstract}

Key Words: Volatile constituents, $\alpha$ Pinene, nonanal, anticonvulsant activity.

\section{Introduction}

The aim of the present study was to identify the volatile constituents and anticonvulcent activities of the aerial parts of Dichrostachys cinerea. D.cinerea belongs to Family Fabaceae the tree is up to 7 meter tall with very characteristic bicoloured flowers (bottlebrush) and is native to South Africa. The plant was used in the traditional Indian system of medicine which is reported to be used in many cases, the bark of the plant is used to treat dysentery, tooth-aches and elephantiasis. The leaves are laxative and used to treat gonorrhoea, boils, stomache problems, can remove poison from snake-bites, as aphrodisiac and as astringent for scorpion bite. Root infusions are taken for leprosy, syphilis coughs, as anthelmentic, purgative and strong diuretic. The plant is used in veterinary medicine in India $^{(1)}$. Joshi and Sharma ${ }^{(2)}$ reported that triterpenoids and some other constituents from Dichrostachys cinerea, Friedelin, friedlan $3 \beta-\mathrm{ol}, \beta-$
\end{abstract}

sitosterol and $\alpha$-amyrin were isolated from the bark of the plant. Heart wood of the plant contained octacosanol and sitosterol, the leaves containe hentricontanol, and $\beta$-amyrin and $\beta$-sitosterol. The phenolic constituents of the aerial parts of the plant were studied, the plant was found to posses good anti-inflammatory, antiulcer and antioxidant activity $^{(3)}$.

\section{Plant material}

\section{Materials}

The aerial parts of $D$. cinerea (leaves, stems and flowers) were obtained from the Orman Botanical Garden, Giza, Egypt. The plant was authenticated by Mrs. Terase Labib, Taxonomist of Orman Garden and confirmed by the Taxonomist, Dr. M. El-Gebaly, National Research Centre (NRC). A voucher specimen (No.135-2003) was kept in the Herbarium of Pharmacognosy Department, NRC. 


\section{Experimental animals}

Adult Sprauge Dawely albino rats weighing 130$150 \mathrm{~g}$ were obtained from the Animal House Colony of the National Research Centre, Egypt. they were kept under the same hygienic conditions and well balanced diet and water. All animal procedures were performed after approval from the ethics committee of the National Research Centre and in accordance with the recommendations for the proper care and use of Laboratory animals (NIH Publication No. 85-23, revised, 1985).

\section{Drugs}

Carbamazepin (Tegretol, Swiss Pharma S.A.E., Cairo, Egypt), was used as a standard anticonvulsant drug.

\section{Apparatus}

1. Gas Chromatograph Coupled with a Mass Spectrometers GC / MS Finnigan mat SSQ 7000, Digital DEC EL eV 70 for GC/MS analysis of volatiles.

2. Modified Likens and Nikerson apparatus was used for preparation of the volatile constituents.

\section{Methods}

\section{Preparation of the volatile constituents}

Three $\mathrm{kg}$ of fresh aerial parts of D.cinerea were subjected to steam distillation in a modified Lickens and Nikerson apparatus ${ }^{(4)}$, which allowed the simultaneous extraction of the volatile components in an organic solvent (n-pentane). The solvent was evaporated carefully after dehydration over anhydrous sodium sulphate. The yielded volatiles were kept in a refrigerator for GC/MS analysis.

\section{Conditions of GC/MS analysis}

Capillary column; DB-5 fused silica (5\% phenyl methylpolysiloxane), $30 \mathrm{~m}$ length, $0.25 \mathrm{~mm}$ id and $0.25 \mu \mathrm{m}$ thickness; Carrier Gas was Helium at 13 psi.; oven temperature was programmed at $60^{\circ} \mathrm{C}$ isothermal for $3 \mathrm{~min}$. then heating to $260^{\circ} \mathrm{C}$ at a rate of $4^{\circ} \mathrm{C} / \mathrm{min}$. then isothermal at $260^{\circ} \mathrm{C}$ for 5 min; Injector Temperature $220{ }^{\circ} \mathrm{C}$; Ionization Energy, 70eV; Volume Injected was $1 \mu 1$.

Identification of the constituents was carried out by comparison of their retention times, and mass spectral fragmentation patterns with those of the available database librarie (Wiley Int. USA) and NIST (Nat. Inst. St. Technol., USA)], and/or published data ${ }^{(1,3)}$. Eight Peaks Index of Mass Spectra $^{(5)}$ and Jennings and Shibamato ${ }^{(6)}$. Quantitative determination was carried out based on peak area integration. The identified compounds of the volatile constituents in Table 1.

\section{B. Investigation of biological activities Anticonvulsant activity test:}

This activity was carried out according to the method reported by Rizzo, et al ${ }^{(7)}$.

Seventy two adult male albino rats, divided into twelve groups, each of six animals were orally treated with two doses $(50,100 \mathrm{mg} / \mathrm{kg}$.bwt $)$ of each extract, carbamazepin (positive control, 100mg/ kg.bwt), or saline (negative control). Electrical stimulation was applied to the rat ear by using 515 Master Shocker (Lafayette Inst. Co.). The increase in voltage required to induce an electrical shock in treated animals is taken as a measure of anticonvulsant activity. Results are compiled in Table 3.

Conflict of Interest statement: The authors are declaring no conflicts of interest.

Table 1: Results of GC/MS analysis of the volatile constituents of the aerial parts of Dichrostachys cinerea $\mathrm{L}$.

\begin{tabular}{|c|l|c|c|c|c|c|}
\hline No. & Compound & RRt & B.P. & $\mathrm{M}^{+}$ & Rel.\% & M. formula \\
\hline 1 & $\alpha$ - Pinene & 1.00 & 93 & 136 & 26.47 & $\mathrm{C}_{10} \mathrm{H}_{16}$ \\
\hline 2 & $\beta$ - Pinene & 1.19 & 93 & 136 & 0.21 & $\mathrm{C}_{10} \mathrm{H}_{16}$ \\
\hline 3 & Myrcene & 1.29 & 93 & 136 & 1.07 & $\mathrm{C}_{10} \mathrm{H}_{16}$ \\
\hline 4 & 2 -Carene & 1.36 & 93 & 136 & 2.54 & $\mathrm{C}_{10} \mathrm{H}_{16}$ \\
\hline 5 & 3 -Carene & 1.49 & 93 & 136 & 1.05 & $\mathrm{C}_{10} \mathrm{H}_{16}$ \\
\hline 6 & cis -Ocimene & 1.54 & 43 & 136 & 0.06 & $\mathrm{C}_{10} \mathrm{H}_{16}$ \\
\hline 7 & $\gamma$ - Terpinene & 1.644 & 43 & 136 & 0.14 & $\mathrm{C}_{10} \mathrm{H}_{16}$ \\
\hline 8 & Terpinolene & 1.805 & 121 & 136 & 0.97 & $\mathrm{C}_{10} \mathrm{H}_{16}$ \\
\hline 9 & n-Undecane & 1.86 & 43 & 156 & 0.16 & $\mathrm{C}_{11} \mathrm{H}_{24}$ \\
\hline 10 & n-Nonanal & 1.97 & 41 & 142 & 5.11 & $\mathrm{C}_{9} \mathrm{H}_{18} \mathrm{O}$ \\
\hline 11 & Orthonitrophenol & 2.10 & 43 & 139 & 0.07 & $\mathrm{C}_{6} \mathrm{H}_{5} \mathrm{O}_{3} \mathrm{~N}$ \\
\hline 12 & trans- Pinocarveol & 2.27 & 41 & 152 & 0.22 & $\mathrm{C}_{10} \mathrm{H}_{16} \mathrm{O}$ \\
\hline 13 & Methylsalicylate & 2.45 & 120 & 152 & 0.98 & $\mathrm{C}_{8} \mathrm{H}_{8} \mathrm{O}_{3}$ \\
\hline
\end{tabular}




\begin{tabular}{|c|c|c|c|c|c|c|}
\hline 14 & n-Dodecane & 2.47 & 43 & 170 & 0.61 & $\mathrm{C}_{12} \mathrm{H}_{26}$ \\
\hline 15 & Pulegone & 2.60 & 41 & 152 & 0.25 & $\mathrm{C}_{10} \mathrm{H}_{16} \mathrm{O}$ \\
\hline 16 & 4-Methyl-2-nitro-phenol & 2.75 & 153 & 153 & 0.12 & $\mathrm{C}_{7} \mathrm{H}_{7} \mathrm{O}_{3} \mathrm{~N}$ \\
\hline 17 & 1-Decanol & 2.81 & 41 & 158 & 0.17 & $\mathrm{C}_{12} \mathrm{H}_{26} \mathrm{O}$ \\
\hline 18 & 5-Methyl dodecane & 2.91 & 43 & 184 & 0.27 & $\mathrm{C}_{13} \mathrm{H}_{28}$ \\
\hline 19 & n-Tridecane & 3.06 & 43 & 184 & 0.13 & $\mathrm{C}_{13} \mathrm{H}_{28}$ \\
\hline 20 & Cumine alcohol & 3.11 & 135 & 150 & 0.47 & $\mathrm{C}_{10} \mathrm{H}_{14} \mathrm{O}$ \\
\hline 21 & 4- Methyl tetradecane & 3.13 & 43 & 212 & 0.49 & $\mathrm{C}_{15} \mathrm{H}_{32}$ \\
\hline 22 & 3-Methoxy benzoic acid & 3.24 & 152 & 152 & 0.48 & $\mathrm{C}_{8} \mathrm{H}_{8} \mathrm{O}_{3}$ \\
\hline 23 & 6-Tridecen-4-yne & 3.27 & 79 & 178 & 0.70 & $\mathrm{C}_{13} \mathrm{H}_{22}$ \\
\hline 24 & $\begin{array}{l}\text { 1,2,3,4-Tetrahydro, } 1,1,6- \\
\text { trimethylnaphthalene }\end{array}$ & 3.31 & 159 & 174 & 0.38 & $\mathrm{C}_{13} \mathrm{H}_{18}$ \\
\hline 25 & $\alpha$-Copaene & 3.43 & 41 & 204 & 0.32 & $\mathrm{C}_{15} \mathrm{H}_{24}$ \\
\hline 26 & 2,4- Dodecadienal & 3.46 & 55 & 180 & 0.15 & $\mathrm{C}_{12} \mathrm{H}_{20} \mathrm{O}$ \\
\hline 27 & cis-Jasmone & 3.56 & 79 & 164 & 0.98 & $\mathrm{C}_{11} \mathrm{H}_{16} \mathrm{O}$ \\
\hline 28 & n-Tetradecane & 3.58 & 57 & 198 & 0.13 & $\mathrm{C}_{14} \mathrm{H}_{3} \mathrm{O}$ \\
\hline 29 & $\beta$-Caryophyllene & 3.71 & 41 & 204 & 1.01 & $\mathrm{C}_{15} \mathrm{H}_{24}$ \\
\hline 30 & $\alpha$-Santalene & 3.72 & 93 & 204 & 0.13 & $\mathrm{C}_{15} \mathrm{H}_{24}$ \\
\hline 31 & Hexylresorcinol & 3.77 & 123 & 194 & 0.13 & $\mathrm{C}_{12} \mathrm{H}_{18} \mathrm{O}_{2}$ \\
\hline 32 & Nerylacetone & 3.86 & 43 & 194 & 0.43 & $\mathrm{C}_{13} \mathrm{H}_{22} \mathrm{O}$ \\
\hline 33 & $\alpha$-Humulene & 3.88 & 93 & 204 & 0.26 & $\mathrm{C}_{15} \mathrm{H}_{24}$ \\
\hline 34 & cis -Methylisoeugenol & 3.90 & 135 & 178 & 0.68 & $\mathrm{C}_{11} \mathrm{H}_{14} \mathrm{O}_{2}$ \\
\hline 35 & $\beta$ - Ionone & 4.02 & 177 & 192 & 1.45 & $\mathrm{C}_{13} \mathrm{H}_{20} \mathrm{O}$ \\
\hline 36 & 2-Methyl tetradecane & 4.04 & 43 & 212 & 0.42 & $\mathrm{C}_{15} \mathrm{H}_{32}$ \\
\hline 37 & Tran- Methylisoeugenol & 4.19 & 178 & 178 & 1.08 & $\mathrm{C}_{11} \mathrm{H}_{14} \mathrm{O}_{2}$ \\
\hline 38 & Tran-Nerolidol & 4.42 & 41 & 222 & 0.25 & $\mathrm{C}_{15} \mathrm{H}_{26} \mathrm{O}$ \\
\hline 39 & Spathulenol & 4.50 & 43 & 220 & 0.20 & $\mathrm{C}_{15} \mathrm{H}_{24} \mathrm{O}$ \\
\hline 40 & n-Hexadecane & 4.56 & 57 & 226 & 0.61 & $\mathrm{C}_{16} \mathrm{H}_{34}$ \\
\hline 41 & Methyl-8(2furyl)octanoate & 4.68 & 81 & 224 & 0.16 & $\mathrm{C}_{13} \mathrm{H}_{20} \mathrm{O}_{3}$ \\
\hline 42 & 1-Tetradecanol & 4.89 & 43 & 214 & 0.15 & $\mathrm{C}_{14} \mathrm{H}_{30} \mathrm{O}$ \\
\hline 43 & 2-Methyl hexadecane & 5.02 & 43 & 240 & 0.40 & $\mathrm{C}_{17} \mathrm{H}_{36}$ \\
\hline 44 & n-Heptadecane & 5.04 & 57 & 240 & 1.31 & $\mathrm{C}_{17} \mathrm{H}_{36}$ \\
\hline 45 & Hexadecanal & 5.07 & 41 & 240 & 0.49 & $\mathrm{C}_{16} \mathrm{H}_{32} \mathrm{O}$ \\
\hline 46 & 1-Pentadecanol & 5.16 & 43 & 228 & 0.11 & $\mathrm{C}_{15} \mathrm{H}_{32} \mathrm{O}$ \\
\hline 47 & 2-Methyl heptatadecane & 5.21 & 43 & 254 & 0.72 & $\mathrm{C}_{18} \mathrm{H}_{38}$ \\
\hline 48 & n-Octadecane & 6.33 & 57 & 254 & 1.45 & $\mathrm{C}_{18} \mathrm{H}_{38}$ \\
\hline 49 & 1-Eicosyne & 5.64 & 43 & 278 & 1.03 & $\mathrm{C}_{20} \mathrm{H}_{38}$ \\
\hline 50 & $\begin{array}{l}6,10,14 \text { Trimethyl-2- } \\
\text { pentadecanone- }\end{array}$ & 5.65 & 43 & 268 & 0.93 & $\mathrm{C}_{18} \mathrm{H}_{36} \mathrm{O}$ \\
\hline 51 & Neophytadiene & 5.66 & 82 & 278 & 0.39 & $\mathrm{C}_{20} \mathrm{H}_{38}$ \\
\hline
\end{tabular}




\begin{tabular}{|c|c|c|c|c|c|c|}
\hline 52 & 9-Eicosyne & 5.81 & 81 & 278 & 0.46 & $\mathrm{C}_{20} \mathrm{H}_{38}$ \\
\hline 53 & $\begin{array}{l}\text { Methyl 8,11,14- } \\
\text { heptadecatrienoate }\end{array}$ & 5.85 & 79 & 278 & 0.79 & $\mathrm{C}_{18} \mathrm{H}_{30} \mathrm{O}_{2}$ \\
\hline 54 & n-Nonadecane & 5.87 & 43 & 268 & 1.05 & $\mathrm{C}_{19} \mathrm{H}_{40}$ \\
\hline 55 & Methylhexadecanoate & 6.00 & 74 & 270 & 0.94 & $\mathrm{C}_{17} \mathrm{H}_{34} \mathrm{O}_{2}$ \\
\hline 56 & Bis(2-ethylbutyl)phthalate & 6.12 & 149 & 334 & 1.59 & $\mathrm{C}_{20} \mathrm{H}_{30} \mathrm{O}_{4}$ \\
\hline 57 & Hexadecanoic acid & 6.30 & 256 & 256 & 2.95 & $\mathrm{C}_{16} \mathrm{H}_{32} \mathrm{O}_{2}$ \\
\hline 58 & n-Eicosane & 6.35 & 57 & 282 & 0.47 & $\mathrm{C}_{20} \mathrm{H}_{42}$ \\
\hline 59 & Methyl-9-octadecenoate & 6.67 & 55 & 296 & 3.78 & $\mathrm{C}_{19} \mathrm{H}_{36} \mathrm{O}_{2}$ \\
\hline 60 & Phytol & 6.78 & 71 & 296 & 1.17 & $\mathrm{C}_{20} \mathrm{H}_{40} \mathrm{O}$ \\
\hline 61 & 8,11,14-Eicosatrienoic acid & 7.00 & 41 & 306 & 4.90 & $\mathrm{C}_{20} \mathrm{H}_{34} \mathrm{O}_{2}$ \\
\hline 62 & n-Docosane & 7.09 & 43 & 310 & 0.88 & $\mathrm{C}_{22} \mathrm{H}_{46}$ \\
\hline 63 & n-Tricosane & 7.43 & 57 & 324 & 1.82 & $\mathrm{C}_{23} \mathrm{H}_{48}$ \\
\hline 64 & n-Tetracosane & 7.77 & 57 & 338 & 1.00 & $\mathrm{C}_{24} \mathrm{H}_{50}$ \\
\hline 65 & n-Pentacosane & 8.09 & 57 & 352 & 1.09 & $\mathrm{C}_{25} \mathrm{H}_{52}$ \\
\hline 66 & Bis(2ethylhexyl)phthalate & 8.21 & 149 & 390 & 1.65 & $\mathrm{C}_{24} \mathrm{H}_{38} \mathrm{O}_{4}$ \\
\hline 67 & n-Hexacosane & 8.41 & 57 & 366 & 0.75 & $\mathrm{C}_{26} \mathrm{H}_{54}$ \\
\hline 68 & n-Heptacosane & 8.75 & 57 & 380 & 1.44 & $\mathrm{C}_{27} \mathrm{H}_{56}$ \\
\hline 69 & n-Octacosane & 9.17 & 57 & 394 & 0.24 & $\mathrm{C}_{28} \mathrm{H}_{58}$ \\
\hline \multirow[t]{3}{*}{70} & Squalene & 9.19 & 69 & 410 & 0.70 & $\mathrm{C}_{30} \mathrm{H}_{50}$ \\
\hline & Total identified constituents & -- & -- & -- & 86.13 & -- \\
\hline & Unidentified constituents & -- & -- & -- & 13.87 & -- \\
\hline
\end{tabular}

$\mathrm{RRt}=$ retention time relative to $\alpha$-Pinene $(\mathrm{Rt}=6.22 \mathrm{~min})$

Table 2: Percentage of different chemical classes in the volatiles of aerial parts of Dichrostachys cinerea $\mathrm{L}$.

\begin{tabular}{|l|c|c|c|}
\hline Chemical class & Relative area percentage & Chemical class & $\begin{array}{l}\text { Relative area } \\
\text { percentage }\end{array}$ \\
\hline I. Oxygenated Compounds & & II. Non-Oxygenated & \\
& 5.409 & Compounds & \\
a. Terpenoids & 23.708 & a. Terpenoids & 34.345 \\
b. Long chain & 3.536 & b. Long chain & 18.595 \\
c. Aromatic & 0.157 & c. Aromatic & 0.380 \\
d. Heterocyclic & 32.81 & & 53.32 \\
& & Total & \\
\hline
\end{tabular}


Table 3: Anticonvulcent activity of total ethanol and successive extracts of the aerial parts Dichrostacys cinerea $\mathrm{L}$.

\begin{tabular}{|c|c|c|c|c|c|c|c|c|}
\hline \multirow[t]{3}{*}{ Group } & \multirow{3}{*}{$\begin{array}{l}\text { Dose (mg lkg } \\
\text { b.wt.) }\end{array}$} & \multirow{3}{*}{$\begin{array}{l}\text { Volts needed } \\
\text { before treatment } \\
\text { (zero time ) }\end{array}$} & \multicolumn{6}{|c|}{ Volts needed after single oral dose } \\
\hline & & & \multicolumn{3}{|c|}{ One hour } & \multicolumn{3}{|c|}{ Two hours } \\
\hline & & & Mean \pm S.E. & \%of change & potency & Mean \pm S.E. & $\%$ of change & potency \\
\hline Control & $1 \mathrm{ml}$ saline & $75.9 \pm 2.6$ & $76.1 \pm 2.3$ & 0.26 & 0.22 & $75.8 \pm 2.1$ & 0.31 & 0.23 \\
\hline Ethanol & $\begin{array}{c}50 \\
100\end{array}$ & $\begin{array}{c}77.1 \pm \\
78.2 \pm 2.4\end{array}$ & $\begin{array}{l}112.8 \pm 5.1 * \\
121.5 \pm 5.6 *\end{array}$ & $\begin{array}{l}46.30 \\
55.37\end{array}$ & $\begin{array}{l}40.34 \\
48.24\end{array}$ & $\begin{array}{l}123.6 \pm 5.3^{*} \\
141.7 \pm 8.1^{*}\end{array}$ & $\begin{array}{l}60.31 \\
81.20\end{array}$ & $\begin{array}{l}43.94 \\
59.16\end{array}$ \\
\hline Pet.ether & $\begin{array}{c}50 \\
100\end{array}$ & $\begin{array}{l}75.1 \pm 1.5 \\
75.1 \pm 2.2\end{array}$ & $\begin{array}{c}98.4 \pm 3.9^{*} \\
120.7 \pm 2.9 *\end{array}$ & $\begin{array}{l}31.02 \\
60.71\end{array}$ & $\begin{array}{l}27.02 . \\
52.90\end{array}$ & $\begin{array}{l}105.7 \pm 3.2^{*} \\
127.8 \pm 3.7 *\end{array}$ & $\begin{array}{l}40.74 \\
70.17\end{array}$ & $\begin{array}{l}29.68 \\
51.12\end{array}$ \\
\hline Chloroform & $\begin{array}{l}50 . \\
100 \\
\end{array}$ & $\begin{array}{l}76.9 \pm 1.8 \\
73.6 \pm 3.1\end{array}$ & $\begin{array}{l}117.3 \pm 4.5 * \\
129.5 \pm 3.2 *\end{array}$ & $\begin{array}{l}52.53 \\
75.95\end{array}$ & $\begin{array}{l}45.76 \\
66.18\end{array}$ & $\begin{array}{l}121.2 \pm 3.6^{*} \\
142.1 \pm 4.8^{*}\end{array}$ & $\begin{array}{l}57.60 \\
93.07\end{array}$ & $\begin{array}{l}41.97 \\
67.81\end{array}$ \\
\hline Ethyl acetate & $\begin{array}{c}50 \\
100\end{array}$ & $\begin{array}{l}77.3 \pm 1.8 \\
72.9 \pm 2.8\end{array}$ & $\begin{array}{c}88.3 \pm 2.9^{*} \\
136.1 \pm 5.6^{*}\end{array}$ & $\begin{array}{l}14.23 \\
86.69\end{array}$ & $\begin{array}{l}12.40 \\
75.53\end{array}$ & $\begin{array}{c}104.2 \pm 5.2^{*} \\
147.1 \pm 3.6^{*}\end{array}$ & $\begin{array}{c}34.80 \\
101.78 \\
\end{array}$ & $\begin{array}{l}25.35 \\
74.15\end{array}$ \\
\hline Methanol & $\begin{array}{l}50 . \\
100\end{array}$ & $\begin{array}{l}73.5 \pm 2.1 \\
74.1 \pm 3.2\end{array}$ & $\begin{array}{l}115.9 \pm 4.8 * \\
131.1 \pm 4.8 *\end{array}$ & $\begin{array}{l}57.69 \\
76.92\end{array}$ & $\begin{array}{l}50.26 \\
67.02\end{array}$ & $\begin{array}{c}118.6 \pm 3.8 * \\
146.4 \pm 4.8 *\end{array}$ & $\begin{array}{l}61.36 \\
97.57\end{array}$ & $\begin{array}{l}44.70 \\
71.09\end{array}$ \\
\hline Carbamazepin & 100 & $76.5 \pm 2.1$ & $164.3 \pm 5.6$ & 114.77 & 100 & $181.5 \pm 6.2 *$ & 137.25 & 100 \\
\hline
\end{tabular}

*Significantly different from zero time at $\mathrm{p}<0.01$

\section{Results and Discussion}

GC/MS analysis of the volatile constituents of $D$. cinerea revealed the identification of 70 compounds representing $(86.13 \%)$ of the total volatiles of the plant. The major compounds were $\alpha$ pinene $(26.47 \%)$, nonanal $(5.109 \%)$ and $8,11,14$ eicosatrienoic acid $(4.898 \%)$.

Oxygenated compounds constituted $(32.81 \%)$ mainly attributed to nonanal(5.109\%), 8,11,14 eicosatrienoic acid (4.898\%), hexadecanoic $\operatorname{acid}(2.954 \%)$, phytol(1.169\%), $\beta$-ionone(1.448\%), methylisoeugenol (1.080\%), methyl salisylate $(0.982 \%)$ and 3-methoxy benzoic acid $(0.477 \%)$.

Non oxygenated compounds represent $53.32 \%$ atributed to $\alpha$ - pinene $(26.47 \%)$, carene $2(2.541 \%)$, tricosane $(1.816 \%)$, heptacosane, (1.444\%), heptadecane $(1.311 \%), \quad$ nonadecane $(1.05 \%)$, carene-3 (1.049\%), caryophyllene $(1.006 \%)$, tetracosane $(1.001 \%)$ and terpinolene $(0.970 \%)$.

GC/MS analysis of the volatile constituents of Dichrostachys cinerea was done for the first time in this study.

The highest anticonvalcent activity was exhibited after 2 hours by100mg of ethyl acetate extract $(74.15 \%$ potency) followed by $100 \mathrm{mg}$ of methanol extract $(71.09 \%)$ followed $100 \mathrm{mg}$ of chloroform extract(67.81\%) and in comparison with $100 \mathrm{mg}$ of carbamazepine (100\% potency).

\section{References}

1. Adams RP. 1989. Identification of Essential Oil by Ion Trap Mass Spectroscopy Academic Press, INC, New York.

2. Joshi KC and Sharma T. Triterpenoids and some other constituents from Dichrostachys cinerea. Phytochemistry. 1974, 13(9), 2010-2011.

3. Adams RP.1995. Identification of Essential Oil Components by Gas Chromatography/Mass Spectroscopy", Allured Publishing Corporation, Carol Stream, Illinois, USA. 4. Macleod AJ and Cave. 'Volatile flavour components of eggs". J. Sci. Food Agric., 1975, 26, 351-360.

5. National Institute of Standards and Technology. 1949. Partridge, SM., Nature, PP. 164

6. Jennings W and Shibamato T. 1981. Qualitative Analysis of Flavor and Fragrance Volatiles by Glass Capillary Gas Chromatography", New York Academic Press.PP.483.

7. Rizzo M, Morselli BL and Jarattini S. 'Further observation on the interaction between phenobarbitone and diphenyl hydantoin drug, chronic trearment in rat . Biochem.Pharmacol, 1971, 21, 449-454. 